\title{
On the wavelength range and quantum efficiency of three-micron laser emission in concentrated Er-systems
}

\section{LUPEI}

Institute of Atomic Physics, 76900 Bucharest, Romania

\begin{abstract}
A generatized model for stationary three-micron laser emission governed by energy transfer processes in concentrated Er-systems is presented. Explicit analytical. equations for the photon flux density in the resonator for a given wavelength is obtained as a function on system parameters lrates of up-conversion nrocesses from the laser levels, efficiency of cross-relaxation processes, cross section of emission, losses, crystal field splitting of levels, lifetimes of laser levels, dopant concentra tion), on pump characteristics (wavelength, intensity) and temperature. These equations describe in a consistent way the existing data on stationary laser emission in concentrated Er systems and suggest possible ways to control and improve the characteristics of emission.
\end{abstract}

The problem of three-micron laser emission between levels $4111 / 2$ and $4113 / 2$ in concentrated Er systems gave rise to controversial debate concernina the mechanism of depopulation of the terminal level whose lifetime is usually much larger than that of the emitting level. Several models based on ESA of pump radiation from $41 / 3 / 2$ and $4 / 11 / 2$ [1], on energy transfer to several rare-earth impurities [2] or on up-conversion process $T_{1}$ from $l_{13 / 2}[3]$,

$$
\left({ }^{4} / 13 / 2+{ }^{4} 1_{13 / 2}\right) \rightarrow\left({ }^{4} / 9 / 2+{ }^{4} / 15 / 2\right)
$$

have been proposed in order to explain the absence of selfsa turation of laser emission. Part of the up-converted excita tion by process (1) could be then reused for laser emission. in order to assure an efficient use of the process (1) a fast energy migration on $4 / 13 / 2$ would be necessary and this re quires in turn high concentration of activator. Unfortunately, at these concentrations, due to the rich energy level diagram of Er ${ }^{3+}$ with many coincidences of the energy gaps, several other energy transfer processes become effective (Figure 1). Acting alone or in combination all these processes reduce the effect of the up-conversion process $T_{1}$. As shown in Figure 1 , these processes involve a large part of the energy levels of 


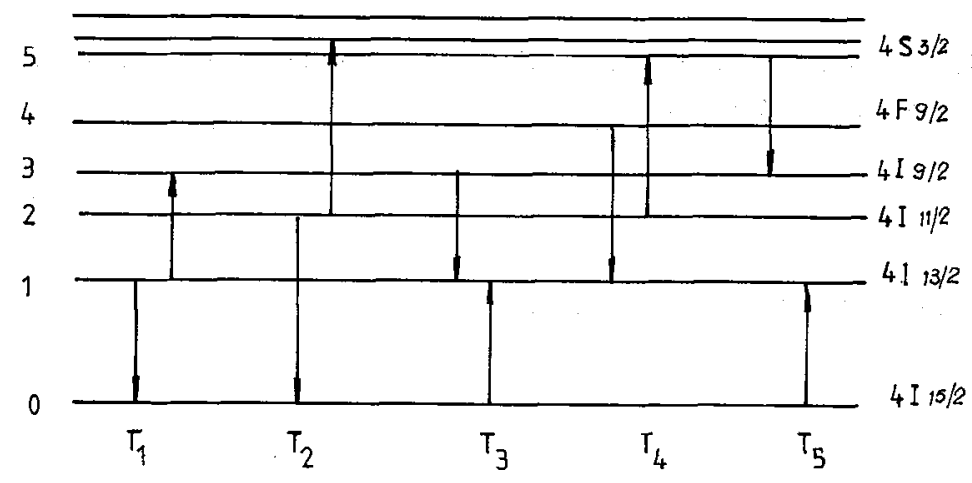

Fig. 1

Er, leading to a unusual circulation of excitation, regardless the pump level. Since the energy pumped or upconverted above the level ${ }^{4} \mathrm{~S}_{3 / 2}$ (thermallized at room temperature with ${ }^{2} \mathrm{H}_{11 / 2}$ ) relaxes usualiy rapldly on this level, we can limit the analy5 is of cross-relaxation processes to those involving levels up to ${ }^{4} \mathrm{~s}_{3 / 2}$;

- the process $T_{2}$ is a up-conversion from $4 / 11 / 2$

$$
\left({ }^{4} 1_{11 / 2}+{ }^{4} 1_{11 / 2}\right) \rightarrow\left({ }^{4} 1_{15 / 2}+{ }^{4}{ }_{5 / 2}\right)
$$

- the process $T_{3}$ is a cross-relaxation

$$
\left({ }^{4} / 9 / 2+{ }^{4} / 15 / 2\right) \rightarrow\left({ }^{4} / 13 / 2+{ }^{4} / 13 / 2\right)
$$

that is in fact an inverse of up-conversion $T_{1}$.

- the process $T$

$$
\left({ }^{4} \mathrm{~F}_{9 / 2} \rightarrow{ }^{4} 1_{13 / 2}\right)+\left({ }^{4} 1_{11 / 2} \rightarrow{ }^{2} H_{11 / 2}\right)
$$

- the process $T_{5}$

$$
\left({ }^{4} s_{3 / 2}+{ }^{4}{ }_{9 / 2}\right)+\left({ }^{4} 1_{15 / 2}+{ }^{4} 1_{13 / 2}\right)
$$

At very high Er concentration several three-ion cross-relaxation or up-conversion processes could become active. The co-doping with other ions could also influence the path of excitation. Thus Cr3+, used usually as a sensitizer has the energy levels $4 \mathrm{~T}$ and $2 \mathrm{E}$ below $4 \mathrm{~S}_{3 / 2}$ (Er) and thus an efficient energy transfer from Er to Cr could take place (process Ts); 
however, the largest part (efficiency $P_{5}$ ) of this energy could be back transferred to the level $4 / 9 / 2$ of Er.

All these energy transfer processes compete with other mechanisms of dezexcitation such as the multiphonon relaxa tion or the radiative emission in determining the evolution of populations of Er levels. Thus, they have an important role in determining the range of the emission transitions that could be used in the laser process.

A rate equation modelling of stationary three micron emission for systems with strong multiphonon relaxation from le vels $4 / 9 / 2$ and $4 F_{9 / 2}$ (i.e. with negligible effect of proces ses $T_{3}$ and $T_{4}$ ), such as $Y A G$ has shown $[4,5]$ that for this regime emission analytical expressions for population inversion and of the photon flux could be obtained and these describe in a consistent way the observed characteristics of the emission (limitation of the wavelength range of emission, the effect of co-doping with $\left(r^{3+}\right)$ by using the energy transfer rates determined from spectroscopic measurements. This paper is an extension of this treatment to systems (such as fluorides) where processes $T_{3}$ and $T_{4}$ are active; it also gives the flux equa tion as explicit function of the emission wavelength enabling a more explicit evidenciation of the effect of various interaction on the characteristics of emission. As in the previous treatment for the cross-relaxation processes dominated by static. donor-acceptor transfer, effective transfer rates are used in the system of rate equations, while for the processes involving rapid migration (the up-conversion processes $T_{1}$ and $\mathrm{T}_{2}$ and cross-relaxation $\mathrm{T}_{3}$ ) the measured rates are employed. By defining the efficiencies of particular energy transfer processes

$$
\begin{aligned}
\ell_{3} & =\frac{P_{3} \tau_{3}}{1+P_{3} \tau_{3}} \\
\ell_{4} & =\frac{P_{4} \tau_{4}}{1+P_{4} \tau_{4}} \\
\ell_{5 a} & =\frac{P_{5} \tau_{5}}{1+\left(P_{5}+P_{5}^{\prime}\right) \tau_{5}} \\
\ell_{5 b} & =\frac{P_{5}^{\tau_{5}}}{1+\left(P_{5}+P_{5}^{\prime}\right) \tau_{5}} \\
\ell_{5 c} & =1-\left(\ell_{5 a}+\ell_{5 b}\right)=\frac{1}{1+\left(P_{5} P_{5}^{\prime}\right) \tau_{5}},
\end{aligned}
$$

where, $P_{i}$ are the effective rates of processes $T_{i}$, the threemicron stationary photon flux density inside the laser resonator for the transition between the crystal field components $j(4 / 11 / 2)$ and $i(4,13 / 2)$, with pump rates $R_{i}$ (in any of the energy levels of $F_{r}$ ) that do not deplete to a sensible extent the ground states is;

$$
\begin{aligned}
\phi_{j i}= & {\left[\left(2 R_{t 2}+T_{t 1}\right)-t_{j i} P_{j i}^{2}\left(R_{t 2} R_{t 1}\right)\right] \frac{n_{0}}{\sigma_{j i}}-\frac{1}{\alpha_{j} \sigma_{j}}\left(\frac{1}{\tau_{2}}+t_{j i} \frac{p_{j i} P_{2}}{\alpha_{j} \sigma_{j i}}\right)-} \\
& -\frac{B_{i}}{\alpha_{j} D_{j i}} n_{1}\left(\frac{1}{\tau_{2}} \frac{2^{p_{j i}} P_{2}}{\alpha_{j} \sigma_{j i}}\right)-\frac{n_{j}}{\tau_{j} D_{j i}}\left(1-t_{j i} P_{j i}^{2}\right)
\end{aligned}
$$


where $\alpha_{j}$ and $\beta_{i}$ are the Boltzmann population factors for the crystal field components $j$ and $i, \sigma_{j} i$ is the cross-section of emission and ji represent the losses at the wavelength of emission; $R_{t 1}$ and $R_{t 2}$ are effective pump rates in levels $41,3 / 2$ and $41,1 / 2$ and they reflect the effect of direct pump and of pump energy transferred to these levels due to crossrelaxation processes:

$$
\begin{aligned}
& R_{t 1}=R_{1}+2 l_{3} R_{3}+\left[2 l_{3}+\frac{1+0_{5 a}-l_{5 b}\left(1-P_{s}\right) l_{4}}{1-l_{4} l_{5 c}}\right] \\
& \left.+\left(1-\ell_{4} \ell_{5 c}\right)^{-1} \ell_{5 a}+\left[2 \ell_{3}+\ell_{4} \ell_{5 c}\left(1-2 l_{3}\right)\right]\left(1-\ell_{5 b}\left(1-P_{5}\right)\right]\right\} R_{5} \\
& R_{t 2}=R_{2}+\left(1-l_{3}\right) R_{3}+\left[\left(1-\ell_{3}\right)-\frac{\left(1-\ell_{3}\right) \ell_{5 b}\left(1-P_{5}\right) l_{4}}{1-l_{4} l_{5 c}}\right] R_{4}+ \\
& +\left[\left(1-\ell_{3}\right)-\frac{\ell_{4} \ell_{5 c}+\left(1-\ell_{3}\right) \ell_{5 b}\left(1-P_{5}\right)}{1-\ell_{4} \ell_{5 c}}\right] R_{5} \text {, }
\end{aligned}
$$

$t$ is a generalized efficiency parameter of the cross-relaxatión processes

$$
t_{j i}=\frac{x}{y+z p_{j i}^{2}}
$$

with

$$
\begin{aligned}
& x=1+\frac{\left[2-\left(1-2 \ell_{3}\right) l_{4} l_{5 c}\right]\left(1-p_{5}\right) l_{5 b}+\left(1-l_{5 a}\right)}{1-\ell_{4} l_{5 c}} \\
& y=1-2_{3} \\
& z=\left(1-l_{3}\right)-\left(1-2 l_{3}\right)\left(1-P_{5}\right) l_{5 b}-\frac{\ell_{5} a^{-2 l_{3} l_{5 b}\left(1-P_{5}\right)}}{1-\ell_{4} \ell_{5 c}}
\end{aligned}
$$

and $p_{j i}$ is a figure of merit of the $j+i$ three-micron laser emission $1 / 2$

$$
p_{j i}=\frac{B_{i}}{a_{j}}\left(\frac{p_{2}}{p_{i}}\right)
$$

Eq. (7) of the photon flux density contains four terms (i) first term, $\phi j i$ is proportional to the pump rates and thus it reflects the effictency of pump. We could thus define a pump quantuum efficiency as the number of photons introduced in the flux by each pump photon

$$
n_{k}=\frac{\left(2 R_{t 2}+R_{t 1}\right)-t_{j i} p_{j i}^{2}\left(R_{t 2}+R_{t 1}\right)}{R_{k}}
$$

or the energy efficiency

$$
n_{e k}=\frac{h v_{j i}}{h v_{k}} k
$$


(ii) the second term $\phi(2)$ is always negative and it is inde pendent of pump; it jithis represents a threshold term; $(i i i)$ the third term $\phi(3)$ is always negative and $i t$ reflects the effect of population of the terminal level coupled with various losses in the system. Since

$$
n_{1} \sim\left[\frac{\left(R_{t 1}+R_{t 2}\right)_{n_{0}}}{p_{1}}\right]^{1 / 2}
$$

the term $\phi_{i}(3)$ is proportional to the square root of the pump rate and thus it reduced the efficiency of the pump leading to an apparent increase of the threshold and to a non-linear dependence of the photon flux on the pump rate; the fourth term $\phi(4)$ is the only one that reflects the effect of lifetime of the terminal level.

Both the first and the fourth term on the right side of eq. (7) could be positive or negative, function of the value of figure of merit $p_{j i}$. Thus $\phi_{j i}$ is positive when

$$
P_{j i}^{2}<\frac{\left(2 R_{t 2}+R_{t 1}\right) y}{x\left(R_{t 2}+R_{t 1}\right)-z\left(2 R_{t 2}+R_{t 1}\right)}
$$

while $\phi_{j i}^{(4)}$ is positive for

$$
p_{j i}^{2}>\frac{y}{x-z} \text {. }
$$

The limiting value of $P_{j} i$ for positive pump effect deDends thus only on the pump wavelength and on the energy transfer processes while only the last determine the condition imposed on $P_{j i}$ for positive $\phi_{j i}$.

According to eq. (I1) the figure of merit $p_{j i}$ depends not only on the ratio $\left(P_{2} / P_{1}\right)$, which is the same for all the transitions between any of the crystal fleld components of $41 / 1 / 2$ and $4 / 13 / 2$ for a given system, but also on the ratio of Boltzmann population factors for the actual crystal field components involved in the laser emission. Thus at a given temperature, each $j \rightarrow i$ transition is characterized by a figure of merit which (and not only the ratio $P_{2} / P_{1}$ plays a crucial role in delineating the transitions for which positive pump effect could be obtained in each particular system at given tem perature in given pump conditions. In fact, a simple relation between this figure of merit and the wavelength of emission could be obtained

$$
\lambda_{j i}=\left(k T \ln \frac{p_{j i}}{B}\right)^{-1}
$$

where

$$
B=\left[[ \operatorname { e x p } ( - \frac { \Delta E _ { j } } { k T } ) ] \left[\left[\exp \left(-\frac{\Delta E_{i}}{k T}\right)^{-1}\left(\frac{P_{2}}{P_{1}}\right)^{1 / 2} \exp \left(-\frac{E_{1}}{k T}\right)\right.\right.\right.
$$

Here $\triangle E_{j}$ and $\Delta E_{i}$ are the energy differences between the crystal field components, $j$ and respective $i$ and the lowest components of levels $4 / 11 / 2$ and $4 / 13 / 2$ and $E_{11}$ is the energy difference between these two lowest energy components. The photon flux density can be then written explicitiy 
as function on the emission wavelength.

$$
\begin{aligned}
\phi_{j i}\left(\lambda_{j l}\right) & =\left[\left(2 R_{t 2}+R_{t 1}\right)-\left(R_{t 2}+R_{t 1}\right) t_{j i} B^{2} \exp \left(\frac{2}{\pi_{j i} k T}\right)\right] \frac{\eta_{0}}{\rho_{j i}}- \\
& -\frac{1}{\alpha_{j} \sigma_{j i}}\left(\frac{1}{\tau_{2}}+t_{j i} \frac{\rho_{j i} P_{2}}{\alpha_{j} \sigma_{j i}}\right)-\frac{B_{i}}{\alpha_{j} \rho_{j i}} \eta_{1}\left(\frac{1}{\tau_{2}}+2 t_{j i} \frac{\rho_{j i} p_{2}}{q_{j} \sigma_{j i}}\right) \\
& -\frac{n_{1}}{\tau_{1} \rho_{j i}}\left[1-t_{j i} B^{2} \exp \left(\frac{2}{\lambda_{j i} k T}\right)\right]
\end{aligned}
$$

The condition for positive pump effect limits the wavelength of emission to

$$
\lambda_{j i}>\frac{2}{k T}\left\{\ln \left[\frac{1}{B^{2}} \frac{\left(2 R_{t 2}+R_{t 1}\right) y}{\left(R_{t 2}+R_{t 1}\right) x-\left(2 R_{t 2}\left(r_{t 1}\right) z\right.}\right]\right\}^{-1}
$$

This shows that the shortest wavelength emission depends not only on the pump conditions and on the efficiencies of crossrelaxation processes but also on temperature (directly and via the parameter B). Evidently, since part of the light flux produced by pump would be lost in compensating the negative terms $\phi(2)$ and $\phi(3)$ (and possibly, $\phi_{j}\left(\begin{array}{l}4 \\ j\end{array}\right)$ ) the lower limit of $\lambda_{j i}$ of the output laser emission would be displaced toward longer wavelengths as compared to the limit given by (19). By contrary, in order to have a positive $\phi(4)$ term the wavelength of emis sion should be shorter than alimit value, i.e.

$\lambda_{j i}<\frac{2}{k T}\left[\ln \left(\frac{1}{B^{2}} \frac{y}{x-y}\right)\right]^{-1}$

According to ea. (18) in a given pumo situation the first to give positive photon flux $\phi_{j} i$ will be the transition with the lowest threshold. Then by increasing the pump intensity, the pump term $\phi(1)$ will overcome the negative terms for other transitions and competition between emission at various wavelengths will be installed whose result will be finally decided in favour of the transition with the highest pump effect.

The form of several parameters in eas. (7) or (18) is greatly simplified for particular cases and a simple and direct analysis of the various experimental data become possible. Among important conclusions resulting from this analysis we mention: - the range of wavelengths of emission at a given temperature for a given system denends on the pump conditions, and for each nump situation (wavelength, intensity) above threshold the emission would be dominated by the transition with the highest oump effect. This explain in a consistent way the modification of emission properties of Er:YLE, when oumping with diode or Ar lasers and, the shift of emission wavelength with pump intensity observed in [1].

- the efficiency of emission at a given wavelength de pends on the pump wavelength, as observed in $[6,7]$.

- important modification of emission characteristics could be obtained by changing the temperature, as observed in $[8]$. 
- co-doping with $\mathrm{Cr}^{3+}$ leads to an extension of the emission wavelength range, as observed in [0], but it could have a positive or negative effect on the efficiency of various emission transitions, function on their figure of merit.

The agreement between the predictions of the model based on depopulation by up-conversion of the terminal laser level $41_{13 / 2}$ for the three-micron emission in concentrated Er-systems and the observed characteristics of laser emission thus confirms the consistency of this model.

\section{References}

1. S.Hubert, D.Meichenin, B.W.Zhou, F. Auzel, J.of Luminesc. $50,7(1991)$.

2. A.A.Kaminskii, T.l.Butaeva, .0. Ivanov, I.V.Mochalov, A.G.Pwt rosyan, G. I.Rogov, V.A. Fedorov, Sov.Tech.Phys. Lett. 2, $308(1976)$.

3. V.1.Zhekov, V.A.Lobachev, T.M.Murina, A.M.Prokhorov, Kvant. Elektron. $11,189^{\prime}(1984)$.

4. V.Lupei, J. de Physique IV, $1, C_{7-395}$ (1991).

5. V.Lupei, S.Georgescu, V.Florea, IEEE J. of Quantum Electronics OE-29, $426(1993)$.

6. R.C.Stoneman, J.G.Lynn, L.Esterowitz, IEEE J.of Quantum Electronics $Q E-28,104 i(i 992)$.

7. B.Schmaul, G. Huber, R.Clausen, B.Chai, P.Likam Wa, M.Bass,

Appl. Phys. Lett. 62, 541 (1993).

8. B.J.Dinerman, P.F.Mouiton, D.M.Rines, Adv. Solid State Lasers 1993 Technical Digest Series, Vol.2, OSA, p.322.

9. G.Huber, E.V.Duczynski, K.Petermann, IEEE 1. of Quantum Electronics $24,920(1988)$. 